\title{
Dilatação térmica: Uma abordagem matemática em física básica universitária
}

\author{
Thermal expansion: A mathematical approach in basic university physics
}

\author{
Paulo Machado Mors*
}

Instituto de Física, Campus do Vale, Universidade Federal do Rio Grande do Sul, Porto Alegre, RS, Brasil

Recebido em 22 de agosto de 2015. Aceito em 30 de setembro de 2015

\begin{abstract}
Comenta-se sobre a oportunidade que o tema da dilatação térmica nos oferece para ressaltar as consequências de aproximações matemáticas no tratamento de um sistema físico simples.

Palavras-chave: dilatação térmica, ensino superior, aproximações matemáticas.
\end{abstract}

It is shown that teaching thermal expansion is a good opportunity to stress the consequences of mathematical approximations when studying a simple physical system.

Keywords: thermal expansion, college teaching, mathematical approximations.

\section{Dilatação térmica: Um comentário ma- temático}

No nível médio de ensino o tema da dilatação térmica é tratado, geralmente, restringindo-se ao que é apresentado nos livros-texto [1,2]. Para o aluno, o assunto não costuma apresentar maiores dificuldades, já que o que lhe é exigido raramente ultrapassa a simples substituição de valores em fórmulas previamente apresentadas. No nível superior de ensino, textos de física universitária, tanto os tradicionais manuais estrangeiros quanto os de autores brasileiros mais consultados, também não levam o assunto muito mais adiante [3-5].

Trazemos, aqui, uma sugestão de tratamento da dilatação térmica que pode servir de motivação para discussões mais instigantes, relacionando um fenômeno físico com seu tratamento matemático usual.

A discussão pode começar com a colocação de um problema clássico, logo depois de apresentados os primeiros conceitos sobre dilatação de sólidos e líquidos. O problema é a tradicional pergunta, encontrada em todos (ou quase todos) os manuais de física térmica básica: dada uma lâmina metálica,

*Endereço de correspondência: mors@if.ufrgs.br se nela é feito um furo circular, depois de aquecida a lâmina, o furo terá aumentado ou diminuído?

Nosso tratamento, aqui, será macroscópico; ou seja, a visão microscópica das interações moleculares não é, de momento, nosso foco.

A solução imediata é apresentada argumentandose que, se o disco metálico retirado ao se perfurar a lâmina sofrer, separadamente, a mesma variação de temperatura que a submetida à lâmina, então se pode concluir que o disco deverá continuar se encaixando perfeitamente no furo, ou seja, terá sofrido a mesma expansão que teria sofrido se nunca tivesse de lá sido retirado (isto é, se o furo não tivesse sido feito). Logo, se a lâmina se expande, então o furo também aumenta.

Levando adiante a discussão, o professor pode sugerir aos alunos que calculem o quanto o furo teve sua área aumentada, em função da variação da temperatura. Como a área do furo é a mesma do disco metálico de lá retirado, quando promovida a mesma variação de temperatura, então trata-se simplesmente de calcular o aumento da superfície do disco. Chamemos de $\alpha$ o coeficiente de dilatação linear ${ }^{1}$ do material de que é feita a lâmina. Se o diâmetro do disco, originalmente, era $d_{0}$, então,

\footnotetext{
${ }^{1}$ Deve-se ter o cuidado de chamar a atenção para o fato de que a expressão coeficiente de dilatação linear refere-se à dilatação
} 
após a variação positiva de temperatura $\Delta \theta$, o novo diâmetro do disco, $d$, será

$$
d=d_{0}(1+\alpha \Delta \theta)
$$

onde estamos trabalhando em região de temperaturas em que, supostamente, o coeficiente de dilatação é mantido constante.

Assim, a área $S$ do disco circular, após a expansão, valerá

$$
S=\pi\left(\frac{d}{2}\right)^{2}=\frac{\pi}{4} d_{0}^{2}(1+\alpha \Delta \theta)^{2},
$$

ou seja, $(1+\alpha \Delta \theta)^{2}$ vezes a área inicial, $S_{0}=\frac{\pi}{4} d_{0}^{2}$ :

$$
S=S_{0}(1+\alpha \Delta \theta)^{2} .
$$

O aluno pode, também, escrever a expressão para a nova área do disco diretamente a partir da relação de dependência do acréscimo de superfície com a variação da temperatura, já que lhe é ensinado que, em analogia com a Eq. (1), a expansão superficial é regida por uma constante de dilatação superficial $\beta$ tal que

$$
\Delta S=S-S_{0}=\beta S_{0} \Delta \theta .
$$

Se estamos trabalhando com um sólido isotrópico, temos então que seu comportamento frente a uma variação de temperatura é o mesmo ao longo de qualquer direção escolhida. Adotando o par ortogonal $x$ - $y$ de eixos coordenados no plano da lâmina concluímos, então, que o coeficiente de dilatação linear ao longo da direção $x$ é o mesmo coeficiente de dilatação linear ao longo do eixo $y$. Logo, $\beta=2 \alpha$ na suposição de um material isotrópicd 2 .

$$
S=S_{0}(1+2 \alpha \Delta \theta) .
$$

Aqui, fica evidente um falso paradoxo, na comparação entre as Eqs. (3) e (5), cujo surgimento o professor pode forçar, ao induzir os alunos a seguirem os dois caminhos de determinação da superfície do disco dilatado. O falso paradoxo se cria quando

ao longo de uma direção espacial, não tendo nenhuma relação com o caráter linear de qualquer expressão.

${ }^{2}$ Essa relação é demonstrável lembrando que, analogamente à definição (7), apresentada logo adiante, para o coeficiente de dilatação linear, tem-se a definição $\beta=\frac{1}{S} \frac{d S}{d \theta}$ para o coeficiente de dilatação superficial. Se tomamos um retângulo do material de lados $\ell_{x}$ e $\ell_{y}$, então $\beta=\frac{1}{\ell_{x} \ell_{y}} \frac{d\left(\ell_{x} \ell_{y}\right)}{d \theta}=$ $\frac{1}{\ell_{x} \ell_{y}}\left[\ell_{x} \frac{d \ell_{y}}{d \theta}+\frac{d \ell x}{d \theta} \ell_{y}\right]=\frac{1}{\ell_{y}} \frac{d \ell_{y}}{d \theta}+\frac{1}{\ell_{x}} \frac{d \ell_{x}}{d \theta}=\alpha_{x}+\alpha_{y}=2 \alpha$, já que, por isotropia, $\alpha_{x}=\alpha_{y}=\alpha$. não se dá a devida importância ao fato de que as expressões (1) e (5) são aproximações, onde termos de ordem superior estão sendo desprezados. Realmente,

$$
(1+\alpha \Delta \theta)^{2} \approx(1+2 \alpha \Delta \theta)
$$

se $\alpha \Delta \theta \ll 1$. Este é sempre o caso, o que torna a questão, na prática, irrelevante. Gaspar [2], Nussenzveig [4] e Chaves [5] chamam a atenção para o fato, quando desprezam termos de ordem superior, mas sem se estenderem em maiores considerações. Isto tem sua importância, se atentarmos para o fato de que nosso propósito, ao ensinar física no início do curso universitário, não é apenas o de fornecer "fórmulas que funcionam na prática". Deixar que os alunos ocupem um tempo discutindo, entre si, qual dos dois resultados é o "melhor", pode ensejar uma rica interação entre eles. Minha experiência é a de que, com muita frequência, dessa discussão acaba saindo a "solução" do falso paradoxo, quando os alunos concluem que a Eq. (6) é justificada, lembrando o binômio de Newton e sua expansão, e as aproximações daí decorrentes quando o segundo termo é muito menor do que a unidade.

A partir daí podemos continuar, apresentando a definição do coeficiente $\alpha$ como sendo

$$
\alpha=\frac{1}{\ell} \frac{d \ell}{d \theta},
$$

a variação relativa do comprimento $\ell$ com a temperatura. O quanto esse coeficiente é, realmente, constante, já é tema de outra discussão. Aqui, estamos considerando constante o coeficiente, o que pode ser válido em faixas relativamente pequenas de temperatura.

Podemos voltar à Eq. (6), chamando a atenção para o fato de que, no limite $\alpha \Delta \theta \rightarrow 0$, os dois lados dessa equação são, efetivamente, iguais

$$
\begin{aligned}
& \lim _{\alpha \Delta \theta \rightarrow 0}(1+\alpha \Delta \theta)^{2}= \\
& \lim _{\alpha \Delta \theta \rightarrow 0}\left[1+2 \alpha \Delta \theta+\alpha^{2}(\Delta \theta)^{2}\right]=(1+2 \alpha \Delta \theta) .
\end{aligned}
$$

Podemos estender a análise, escrevendo a Eq. (7) como $d \ell=\alpha \ell d \theta$. Integrando:

$$
\int_{i}^{f} d \ell=\int_{i}^{f} \alpha \ell d \theta \Rightarrow \Delta \ell=\alpha \int_{i}^{f} \ell d \theta
$$

onde $i$ e $f$ referem-se ao início e ao final do processo de aquecimento, respectivamente. Este resultado pode ser escrito como 


$$
\Delta \ell=\alpha \bar{\ell} \Delta \theta
$$

com $\bar{\ell}$ representando o valor médio (em temperatura) do comprimento $\ell$ durante o processo. Lembremos que $\Delta \ell=\ell_{f}-\ell_{i}$ e que $\Delta \theta=\theta_{f}-\theta_{i}$. Assim, se adotamos como aproximação a substituição de $\bar{\ell}$ por $\ell_{i}$, então obtemos a expressão usual

$$
\ell_{f}=\ell_{i}(1+\alpha \Delta \theta),
$$

que foi utilizada para escrever a Eq. (1). A Eq. (10) é uma linearização da Eq. (9), muito conveniente e justificável, dados os valores com que normalmente trabalhamos. Portanto, não há por que evitar a menção clara do caminho adotado. Esta certamente é uma boa oportunidade de se falar em aproximações e seus limites de validade.

Se a turma de universitários estiver suficientemente motivada, pode-se comentar que a Eq. (7) é uma equação diferencial, como muitas que eles encontrarão estudando física, esta de facílima solução.

O professor pode mostrar, por substituição, que a solução real mais geral para a Eq. (7) é uma exponencial,

$$
\ell(\theta)=\ell_{0} e^{\alpha\left(\theta-\theta_{0}\right)},
$$

$\operatorname{com} \theta=\theta_{f}, \theta_{0}=\theta_{i}, \ell_{0}$ sendo o comprimento inicial $\ell\left(\theta_{0}\right)$. Lembrando aos alunos a expansão da exponencial em série de potências,

$$
e^{x}=1+x+\frac{x^{2}}{2 !}+\frac{x^{3}}{3 !}+\ldots,
$$

a linearização da solução (11) é imediata: se $\alpha(\theta-$ $\left.\theta_{0}\right)=\alpha \Delta \theta \ll 1$ (o que costuma ser o caso), torna-se válida a aproximação $\ell(\theta)=\ell_{0}(1+\alpha \Delta \theta)$, equivalente à Eq. (10).

Resta-nos mencionar alguns tópicos que merecem, a critério do professor, ser mencionados por ocasião do tratamento acima exposto. Um tópico digno de nota é o tratamento da dilatação volumétrica de fluidos isotrópicos, com uma definição análoga à Eq. (7). Na termodinâmica, é usual definir-se, para um gás, o coeficiente de dilatação

$$
\gamma_{\theta}=\frac{1}{V}\left(\frac{\partial V}{\partial \theta}\right)_{p, N} .
$$

Na Eq. (13), $V$ é o volume do gás, $p$ é sua pressão e $N$ é o número de moles constituindo uma amostra. A medida da variação do volume com a temperatura, aqui, é apresentada através de uma derivada parcial, isto é, a derivada de uma função de várias variáveis em relação a uma dessas variáveis. Para funções de uma variável, $f(x)$, por exemplo, a derivação é denotada por $\mathrm{d} f / \mathrm{d} x$. No caso de uma função de várias variáveis, seja $f(x, y)$, denota-se a derivada parcial de $f$ em relação a $x$, por exemplo, por $\partial f / \partial x$. Os índices $p, N$ junto ao operador indicam que, para a medida do coeficiente de dilatação, mede-se a variação do volume com a temperatura, mantendose constantes as demais variáveis.

Outro tópico relacionado é a dilatação térmica da água que, contrariamente aos casos em que a linearização é uma boa aproximação, com coeficientes de dilatação positivos - perfis lineares de volume versus temperatura com inclinação constante -, apresenta um comportamento anômalo entre as temperaturas de 0 e 4 graus Celsius. Nesta faixa de temperaturas, na transição da estrutura cristalina do gelo para a fase líquida sem estrutura cristalina definida, a curva volume versus temperatura decai, e, portanto, tem-se aí uma região de coeficiente de dilatação volumétrica não constante e negativo.

Livros didáticos de física térmica apresentam tabelas de valores numéricos de coeficientes de expansão térmica. Nessas, pode-se observar que os líquidos apresentam coeficientes de expansão da ordem de cem vezes maiores que os dos sólidos. Ademais, fato muito importante no estudo dos gases, estes possuem coeficientes muito próximos uns dos outros. Valores de coeficientes de expansão podem ser encontrados na página eletrônica WebCalc - Calculadoras On-Line [6].

Procuramos, aqui, mostrar que um assunto simples e muitas vezes subestimado em seu conteúdo, como é o caso do estudo da dilatação térmica, pode ensejar uma abordagem estimulante e esclarecedora sobre aproximações, em física, e o papel da matemática em seu tratamento.

O conteúdo deste trabalho foi enriquecido a partir de comentários feitos pelo árbitro, a quem o autor agradece.

\section{Referências}

[1] A. Máximo e B. Alvarenga, Curso de Física (Scipione, São Paulo, 2006), v. 2.

[2] A. Gaspar, Física (Ática, São Paulo, 2003), v. 2.

[3] P.A. Tipler e G. Mosca, Física para Cientistas e Engenheiros (LTC, Rio de Janeiro, 2009, 6. ed.), v. 1 . 
[4] H.M. Nussenzveig, Curso de Física Básica (Edgar Blücher, São Paulo, 1983), v. 2.

[5] A. Chaves, Física (Reichmann \& Affonso, Rio de Janeiro, 2001), v. 4.

[6] http://www.webcalc.com.br/engenharia/ dilat_alfa.html e http://www.webcalc.com. br/engenharia/dilat_gama.html, conforme consultadas em 22 de setembro de 2015 em http://www.webcalc.com.br/ 